\title{
"Identify-to-reject": A specific strategy to avoid false memories in the DRM paradigm
}

\author{
Paula Carneiro • Angel Fernandez $\cdot$ Emiliano Diez • \\ Leonel Garcia-Marques • Tânia Ramos • \\ Mário B. Ferreira
}

Published online: 5 October 2011

(C) Psychonomic Society, Inc. 2011

\begin{abstract}
Previous research using the Deese-RoedigerMcDermott (DRM) paradigm has shown that lists of associates in which the critical words were easily identified as the themes of the lists produce lower levels of false memories in adults. In an attempt to analyze whether this effect is due to the application of a specific memory-editing process (the identify-to-reject strategy), two experiments manipulated variables that are likely to disrupt this strategy either at encoding or at retrieval. In Experiment 1, lists were presented at a very fast presentation rate to reduce the possibility of identifying the missing critical word as the theme of the list, and in Experiment 2, participants were pressed to give yes/no recognition answers within a very short time. The results showed that both of these manipulations disrupted the identifiability effect, indicating that the identifyto-reject strategy and theme identifiability play a major role in the rejection of false memories in the DRM paradigm.
\end{abstract}

Keywords False memories · DRM paradigm · Memory editing $\cdot$ Theme identifiability Identify-to-reject

Analyzing how to avoid false memories can be a way of better understanding the mechanisms that stand behind their

P. Carneiro • L. Garcia-Marques • T. Ramos • M. B. Ferreira

Faculty of Psychology, University of Lisbon,

Lisbon, Portugal

A. Fernandez $\cdot$ E. Diez

Faculty of Psychology, University of Salamanca,

Salamanca, Spain

P. Carneiro $(\bowtie)$

Faculdade de Psicologia, Alameda da Universidade, 1649-013 Lisboa, Portugal

e-mail: mpcarneiro@fp.ul.pt formation. The majority of research in this field has been centered on the rates of false recall and/or false recognition but has not always been concerned with the effort that the participants put in when avoiding false memories. And, without taking this effort into account, it is difficult to know whether the level of false memories produced is merely the result of error inflation processes or is also modulated by the intervention of some kind of memory rejection mechanisms. Moreover, the absence of a false memory following customary manipulations could mean either that the characteristics of the task did not lead to error inflation or that its production was successfully avoided by the participants. In the present study, we tried to disentangle the contribution of these processes by creating conditions under which participants could intentionally avoid false memories, by using one specific memory-editing strategy and conditions that did not facilitate the application of such a strategy. As will be shown, this particular strategy (identifyto-reject) seems to be specifically used in associative converging tasks and depends on the thematic extraction of the information. We present two experiments indicating that theme identifiability is a variable that greatly contributes to the rejection of false memories by promoting the use of the identify-to-reject strategy.

\section{Memory-editing mechanisms}

There are different ways by which participants may avoid false memories. Recall-to-reject and the use of a distinctiveness heuristic are two special editing mechanisms that participants can employ to achieve this goal. In general, the term recall-to-reject is used to describe the process by which, in recognition tasks, test probes that are similar to studied items are rejected because participants recall a 
specific studied item, which allows them to better detect a mismatch on some of the features. Thus, false memories can be avoided by specifically recalling true events that facilitate the rejection of other, similar items. This process is more easily engaged when the recall of the item in one list or context is mutually exclusive with its occurrence in another list (Jacoby, 1991; Rotello \& Heit, 2000) or context (McElree, Dolan, \& Jacoby, 1999).

Fuzzy trace theory has also described a similar process, recollection rejection, to account for the same phenomenon (Brainerd, Reyna, Wright, \& Mojardin, 2003). In this dualprocess conception of false memory (Brainerd \& Reyna, 2005), for every memorial event, two independent memory traces are encoded: a gist trace that captures the underlying meaning of the event, and a verbatim trace corresponding to the exact representation of the surface features of the event. According to fuzzy trace theory, recollection rejection occurs when a test probe evokes the retrieval of the verbatim memory trace of a studied item and the comparison between the probe and the trace result in a mismatch. For example, participants could remember that the word couch, but not sofa, was previously presented because the studied list did not contain semantically related words. Even if this is not the case, recollection rejection can also occur when the probe and list words, even if sharing the semantic meaning, still differ in noticeable surface features, such as orthography and phonology. It is worth noting that fuzzy trace theory holds that recollection rejection could as well occur on a more intuitive basis or by means of an attributional process, as when participants are aware that the test probe familiarity can be attributed to similarity with a studied item (e.g., "Sofa probably just seems familiar because couch was presented, so I'll reject sofa').

Another way to edit false memories is through the use of a distinctiveness heuristic. Schacter and collaborators (Dodson \& Schacter, 2001, 2002; Schacter, Cendan, Dodson, \& Clifford, 2001; Schacter, Israel, \& Racine, 1999) showed that participants were able to edit false memories on the basis of how vivid and distinctive they expected their memories to be. This strategy involves the comparison between the distinctiveness of the test probes and the distinctiveness of the presented items, together with the expectation that, in order to be considered a presented item, the memory of the probe should be as vivid and distinctive as that of the studied items. If the memory of the probe is less clear and vivid, it is probably because the item is new. Consistently, it has been shown that when the items are presented for study in a perceptually distinctive format, such as pictures, fewer false memories are produced, allegedly because participants can use the following criterion at test: "If I do not remember seeing a picture of an item, it is probably new" (Schacter et al., 1999). The distinctiveness heuristic can also account for cases in which test probes are rejected because, instead of lacking in distinctiveness, they are actually more distinctive than the studied words (e.g., Madigan \& Neuse, 2004; Pesta, Murphy, \& Sanders, 2001). For example, one's own name is rarely falsely recognized in a list composed of common names (Brown, Lewis, \& Monk, 1977). In this case, the participants could use the following criterion: "If my name was presented, I would remember it." In either case, participants reject previously unpresented probes on the basis of the absence of an expected recollection.

These two ways of avoiding false memories have been recently reconceptualized by Gallo (2006) within a sourcemonitoring framework. From this integrative perspective, there are two types of monitoring that can be used, disqualifying monitoring and diagnostic monitoring, each corresponding to different decision processes that can be used to avoid false memories: Disqualifying monitoring, similar to the recall-to-reject process, is based on a true recollection of one item or event, whereas diagnostic monitoring, similar to the distinctiveness heuristic, is based on the absence of an expected recollection.

Although these different mechanisms of false memory avoidance can, in principle, be applied to any kind of false memory paradigm, it seems that some rejection mechanisms are more frequent in some paradigms than in others. For the purpose of this research, we are especially interested in the rejection mechanisms used in the DeeseRoediger-McDermott (DRM) paradigm. This paradigm involves presenting study lists of related words that are to be later recalled or recognized. Each list has the particularity of being composed by the stronger associates of a notpresented converging word (the critical item), the memory of which will be the main measure of interest on subsequent memory tests. The seminal studies (Deese, 1959; Roediger \& McDermott, 1995) found that these critical items could be erroneously recalled or recognized with more likelihood than some of the words that were actually presented, a finding that has been replicated in a vast number of experiments in recent years (see Gallo, 2010, for a review of 15 years of fruitful research with the DRM paradigm).

Lampinen, Meier, Arnal, and Leding (2005) have analyzed the different memory-editing mechanisms used in this paradigm, applying a think-out-loud procedure, and they concluded that different mechanisms are used for different kinds of unstudied items: Critical items are edited mainly by means of recall-to-reject or recollection-rejection strategies, whereas unrelated distractor items are edited mainly through the use of a distinctiveness heuristic. According to this interpretation, recollection rejection requires that one negate the likelihood that a related item was presented by recollecting an item's true presentation. Being the critical words related to studied items, participants rely on the contrasting evidence between studied and 
critical words in order to correctly reject the critical words. In contrast, for unrelated items, it is easier to use lack of distinctiveness as a cue to decide that the item was probably not presented, without the need of a recollection-rejection mechanism.

\section{The role of theme identifiability on the rejection of false memories}

Taking into account that the converging-associate nature of DRM study materials often results in the creation of lists that possess a certain thematic structure, we would like to advance the hypothesis that there is one specific memoryediting mechanism that is particularly relevant in reducing memory distortions in this paradigm. In our previous work (Carneiro, Fernandez, \& Dias, 2009), we showed that lists of associates in which the critical words were easily identified as the themes of the lists produced lower levels of false memories in adults. A similar result was obtained in another study (Neuschatz, Benoit, \& Payne, 2003) when the participants were warned about the DRM phenomenon before they studied the lists. We previously suggested that this result was due to the application of one specific editing strategy, the identify-to-reject strategy. Because the DRM lists are composed of associates that all converge onto a critical word, we predicted that the identification of this missing word could be used to later reject it, thus avoiding false memories. This specific strategy was described by Gallo (2006) as a specific case of the recall-to-reject mechanism, very obvious when the participants had been forewarned about the false memory effect (Gallo, Roberts, \& Seamon, 1997). Other studies have also suggested that participants might use a strategy of this kind even when they are not warned about the false memory effect (Brédart, 2000; Mukai, 2005). However, it is worth mentioning that this specific mechanism has not been taken into consideration in the scarce studies that have tried to discriminate between the diferent memory-editing mechanisms usually used in DRM studies (e.g., Lampinen et al., 2005).

To summarize, when memorizing a DRM list, participants may employ strategic processes that lead to the identification of the critical items during encoding of the study items and then mentally tag them as "not presented." The finding that higher identifiability of critical words leads to lower levels of false memories seems to indicate that participants are using this type of memory-editing strategy, but direct evidence that, in this case, the participants are actually using a memoryediting mechanism has never been shown. Searching for that kind of evidence is the main goal of this study, an effort to show that the identifiability effect is due to the application of a memory-editing mechanism that crucially relies on the identification of the theme and its later rejection to counter false memory errors. Previous research on DRM has almost never mentioned this specific strategy as one possible way to reject false memories, and it has never differentiated it within the more vast disqualifying monitoring set of processes (Gallo, 2006). However, it should be noted that this strategy seems to rely not on verbatim traces of studied items but, instead, on true recall of a particular aspect of participants' cognitive context at the time of study - specifically, participants' inferences about the theme of the lists. In support of this interpretation, the study by Carneiro et al. (2009) found that theme identifiability did not have an effect on veridical memory.

Clarifying the issues regarding the identificability effect and the ways in which it can lead to successfully editing out false memories can have theoretical implications. We have previously argued (Carneiro et al., 2009) that it would be very difficult for fuzzy trace theory to accommodate the finding that greater thematic or gist identification leads to fewer false memories. However, we now acknowledge that the manipulation of theme identifiability can be considered as a direct manipulation of gist (Cann, McRae, \& Katz, 2011) and, therefore, can even provide a particularly appropriate test for this theoretical approach. The reason for our previous contention was, of course, that fuzzy trace theory predicts that gist extraction is responsible for false memories. As such, from a fuzzy trace theory perspective, ease of gist extraction would lead to false memory inflation in thematic lists, not to the opposite pattern that we have obtained (Carneiro et al., 2009). And in fact, there is vast evidence showing that increasing semantic processing usually enhances the DRM illusion. False memories increased when manipulations that strengthen gist identification/extraction-such as, for example, blocking the studied words (McDermott, 1996), deep levels of processing (Thapar \& McDermott, 2001), or relational processing (McCabe, Presmanes, Robertson, \& Smith, 2004)-were used. Yet, as a dual-process theory, fuzzy trace theory also incorporates an editing process that could suppress false memories, usually by recollecting information about the originally studied items. Therefore, in this case, the fact that the critical items came to mind during study can serve as the basis of recollection rejection; then the identify-to-reject strategy can be viewed as one specific case of this memoryediting process. Since the emergence of a false memory reflects a balance between the inflated and the editing processes, it is likely that gist identification affects both processes. Thus, when gist identification is easy, gist extraction will lead to fewer false memories, but when gist identification is hard, false memories will even be higher than in standard conditions. Thus, the explanation of this finding by fuzzy trace theory requires that (1) conditions that prevent the action of recollection rejection will lead to more false memories for lists with stronger gist (high- 
identifiable lists), and (2) conditions that allow the action of recollection rejection, where the identify-to-reject strategy could be viewed as one specific case, will lead to fewer false memories for lists with stronger gist.

To analyze whether the identifiability effect is the result of the operation of a memory-editing mechanism - specifically, the identify-to-reject strategy — we manipulated variables that were likely to disrupt this strategy. On the assumption that this strategy is dependent on theme identification at encoding, our hypothesis was that any manipulation that substantially reduces the possibility of identifying the missing critical word as the unpresented theme of the list during list presentation will eliminate the identifiability effect. Furthermore, because this editing strategy is also dependent on the availability of processing resources at the time of retrieval, such as time to use a recollection-based decision process, it was predicted that a manipulation that severely limited the time allowed for answering in a recognition test would also contribute to the elimination of the effect. In Experiment 1, associative lists of low and high identifiability were presented at a very fast presentation rate, with the aim of impairing, at encoding, the participants' ability to notice that the theme word was not actually presented on the list. In Experiment 2, participants were pressed to give their recognition answers within a very short time, with the aim of reducing, at retrieval, the possibility of using any type of memory-editing mechanism that would require a relatively extended period of time to be effectively applied. As was noted above, both of these manipulations, one operating at encoding and the other at retrieval, were expected to significantly reduce or even eliminate the possibility of using the identify-to-reject strategy.

\section{Experiment 1}

In the first experiment, the rate of list presentation was manipulated at study. McDermott and Watson (2001) studied this variable within the DRM paradigm, varying exposure of items at study from 20 to $5,000 \mathrm{~ms}$, and showed that the effect of presentation rate on false memories followed a nonmonotonic function - that is, an inverted U-shaped relation. This means that slowing the presentation rate increases false recall until a certain point (around $250 \mathrm{~ms}$ ), after which it progressively decreases. The decrement in false recall with a slowing presentation rate has been generally interpreted as a result of the action of monitoring strategies. Thus, Gallo (2006) argued that this false-memory reduction effect could reflect any one of the two types of monitoring processes. It could be interpreted as diagnostic monitoring because, having more time to engage in item-specific processing of list items, participants could produce highly distinctive recollections of the presented items. It could also be interpreted as disqualifying monitoring because, with more time at encoding, true recall would be high and recall of true items would allow participants to discard critical words.

Because the identify-to-reject strategy is dependent on theme identification, it is expected that any encoding variable that makes it difficult for the participants to be aware of the absence of the critical word in the presented list will tend to eliminate the identifiability effect. Therefore, in conditions in which the words are presented very quickly, participants are not able to fully read all the presented words in the list, and, as a result, they cannot be sure whether the theme word was in fact presented or not. Hence, the participants are unable to tag any related word as unpresented, because they are aware that many other words were presented but they cannot visualize all of them.

In the present experiment, we manipulated presentation rates as a between-subjects variable, with three different exposure times of list items at study: the standard rate (2,000 ms), a condition in which we expected to replicate the identifiability effect (i.e., lower levels of false memories for high-identifiable lists than for low-identifiable lists); a very fast rate $(50 \mathrm{~ms})$, with which we expected to eliminate the effect; and an intermediate rate $(250 \mathrm{~ms})$, with which we expected to obtain a pattern that stood between the other two rates. The 50ms duration was chosen as the very fast presentation rate that would disrupt the processing of the to-be-remembered words, while still allowing the extraction of the theme or gist of the list before processing of the presented items was completed (see corroborating arguments in Brainerd, Wright, Reyna, \& Payne, 2002). However, and because participants at this rate cannot visualize all the words in the lists, they cannot keep track of whether the theme word was, in fact, presented or not and are not able to identify that word as a missing word. Consequently, and consistent with the results of previous studies on presentation rates (e.g., McDermott \& Watson, 2001; Seamon, Luo \& Gallo, 1998), we expected that, at this fast rate, false memories would be reliably produced. Also, and because it is assumed that at these short study times the information needed for false memory rejection cannot be gathered, we expected to find higher levels of false memories in this condition than in the other conditions. In line with the arguments presented above, fuzzy trace theory predicts higher false memories for lists with stronger gist (high-identifiable lists), as compared with lists with weak gist (low-identifiable lists), in a condition with a very fast presentation rate where memory editing is prevented, and the opposite pattern for a standard presentation rate.

\section{Method}

Participants Seventy-five university students from Lisbon University took part in this experiment for course credit. All 
were native Portuguese speakers, mainly female (65 of them female; mean age $=22$ years), and all were taking psychology courses. The participants' ages did not differ significantly between the three conditions.

Design The experiment followed a 2 (critical-word identifiability: high vs. low) $\times 3$ (presentation rate: 50 vs. 250 vs. $2,000 \mathrm{~ms}$ ) factorial design, with repeated measures over the first factor.

Materials The associative lists used in this study were selected according to the identifiability scores of the normative study by Carneiro et al. (2009). In that normative study, participants were presented with several DRM lists and were asked to generate, for each list, a single word that best defined its overall theme. Theme identifiability of each list was given by the percentage of participants who produced the critical word as the theme of the list. Twelve of these associative Portuguese lists that produced extreme levels of critical-word identifiability were used in this experiment. Six lists were of high identifiability (critical words: cinema, clown, cow, lion, music, and spider), with percentages of theme identification ranging from 64.6 to 76.8. The other six lists were of low identifiability (critical words: cold, high, hour, kiss, pen, and wheel), with percentages of theme identification ranging from 1.1 to 15. The lists consisted of the 15 strongest associates of each critical word, arranged in decreasing order of strength. The critical words on the high- and low-identifiability lists did not differ in frequency in the language (Nascimento, Casteleiro, Marques, Barreto, \& Amaro, n.d.), length, or mean strength of forward association (Albuquerque, 2001).

In order to control for potential confounding effects by other linguistic characteristics that could be differentially distributed between the two types of lists, and in the absence of relevant data in Portuguese, additional data, involving 345 new participants, were collected for the present study. The characteristics and relevance of the new data are described next, with quantitative estimates and results of statistical tests presented in Table 1. First of all, free association norms for all 180 words in these 12 lists were collected, ${ }^{1}$ so that the mean strength of backward association (MBAS) could be determined for each list in relation to its corresponding critical item. These new data

\footnotetext{
${ }^{1}$ To collect discrete free association norms, we followed the instructions in Nelson, McEvoy, and Schreiber (2004). One hundred eighty words (all the items of the 12 lists) were the target stimuli, and they were presented in a random order mixed within a large set of words, in order to present participants with a more heterogeneous pool of cues. Sixteen different versions of booklets were constructed to control for order effects and also to avoid contamination by the processing of related cues (only three words per list were included in each version). Each word was tested by approximately 90 participants.
}

Table 1 Mean scores of semantic dimensions (scale from 1 to 7), mean occurrences of knowledge types, and mean strength of backward association for lists of high and low identifiability

\begin{tabular}{|c|c|c|c|c|c|c|}
\hline & \multicolumn{2}{|c|}{ High Ident. } & \multicolumn{2}{|c|}{ Low Ident. } & \multirow[t]{2}{*}{$t$} & \multirow[t]{2}{*}{$p$} \\
\hline & $M$ & $S D$ & $M$ & $S D$ & & \\
\hline \multicolumn{7}{|c|}{ Toglia and Battig (1978) dimensions } \\
\hline familiarity & 4.90 & 0.98 & 5.67 & 0.87 & 1.44 & 0.18 \\
\hline meaningfulness & 4.22 & 0.89 & 3.89 & 0.71 & 0.70 & 0.50 \\
\hline concreteness & 5.88 & 0.70 & 4.87 & 0.99 & 2.03 & 0.07 \\
\hline imagery & 5.61 & 0.36 & 4.79 & 1.12 & 1.71 & 0.14 \\
\hline \multicolumn{7}{|c|}{ Wu and Barsalou (2009) knowledge types } \\
\hline situation features & 6.17 & 3.76 & 5.00 & 2.83 & 0.61 & 0.56 \\
\hline synonyms & 0.17 & 0.41 & 0.50 & 0.55 & 1.20 & 0.26 \\
\hline taxonomic relations & 3.17 & 1.60 & 3.50 & 1.87 & 0.33 & 0.75 \\
\hline BAS & 0.15 & 0.07 & 0.11 & 0.08 & 0.92 & 0.38 \\
\hline
\end{tabular}

permitted a very important control, since BAS is considered to be a main predictor of false memory (initially demonstrated by Deese, 1959, and more recently by Roediger, Watson, McDermott, \& Gallo, 2001). The results of a statistical comparison showed that MBAS did not significantly differ in lists of high and low identifiability. Second, empirically derived values for several semantic dimensions of the critical items were obtained. ${ }^{2}$ A study by Brainerd, Yang, Reyna, Howe, and Mills (2008) showed that both the rate of false memories and variation in BAS were consistently related to some semantic characteristics of the critical items. Thus, for the present study, values for familiarity and meaningfulness of the 12 critical items were obtained, since they were the two dimensions that contributed most to false recall and recognition in Brainerd et al. (2008); additionally, their concreteness and imagery values were obtained, because they have been shown to be related to variability in true recall (which could indirectly affect false memories) by Brainerd et al. (2008) and to variability in false memories by Pérez-Mata, Read, and Diges (2002). The analyses showed that the critical words on the highand low-identifialbility lists did not significantly differ in any of these variables (only in the case of concreteness did the difference approach statistical significance). Finally, the knowledge type taxonomy of $\mathrm{Wu}$ and Barsalou (2009) was used to classify semantic relations in the 12 lists, since previous research by Cann et al. (2011) has shown that some of those relationships can explain false recall.

\footnotetext{
${ }^{2}$ To collect the semantic dimensions of the critical items, we followed the instructions in the Toglia and Battig (1978) study. The target stimuli (12 critical words) were disposed in random order among other unrelated words, and eight different versions of booklets were constructed to control for order effects. Approximately 80 different participants were tested in each semantic dimension.
} 
Following their procedure and scoring rubric, two independent raters coded the semantic relations between each of the list words and their corresponding critical word (with an agreement rate of $100 \%$ after disagreements were discussed, as was done in the Can et al., 2011, study) in regard to situation features, synonymy, and taxonomic relation, which were the three knowledge types that contributed most to false recall in the study by Cann et al. Again, no significant differences were found between the scores assigned to the high- and low-identifiability lists.

The final recognition test was composed of 72 words: the critical words of the 12 presented lists, 2 studied words per list (positions 1 and 11), and the critical words and 2 list words (positions 1 and 11) from 12 other unpresented, similarly structured lists that served as unrelated distractors in the test.

Procedure The participants were randomly assigned to one of the three group conditions: 50-, 250-, or 2,000$\mathrm{ms}$ presentation rate. Although the words were presented at different rates across the three conditions, the interstimulus interval was held constant (always $30 \mathrm{~ms}$ ). The sequence of experimental events was controlled via a custom-made computer program using E-Prime software, with the instructions used in the McDermott and Watson (2001) study. In all the conditions, the participants were instructed to memorize the presented lists words for an immediate recall task. The 12 lists (6 of high and 6 of low identifiability) were randomly presented, and after each list, the participants had $1.5 \mathrm{~min}$ to recall the words from that list. A booklet of 12 blank pages was given in advance to each participant to perform this recall task, and they were asked to write down the recalled words from each list on a separate page. In all conditions, they were instructed to avoid guessing. In the fast (250-ms) and very fast (50-ms) presentation conditions, they were advised that the lists were going to be presented quickly $(250 \mathrm{~ms})$ or very quickly $(50 \mathrm{~ms})$, respectively. In the very fast presentation condition, the participants were also informed that due to the speed of presentation, reading of the entire list was not possible, so they should report only the words they could see.

Following recall of the last list, a recognition test was administered, with the words presented visually, one by one, on the computer screen and the responses given on the computer keyboard. The instructions for the recognition task followed standard instructions, similar in the three conditions. The participants had to press one of the two keys ("yes" or "no") for "word previously presented" and "word not presented," respectively. It was administered as a self-paced task, in which the test words remained on the screen until the yes/no response key was pressed.
Results

Recall task Figure 1 displays the proportion of studied and critical words recalled in each presentation rate condition (50, 250, and 2,000 ms) for both types of lists (low and high identifiability). As can be observed, veridical recall tended to linearly increase with exposure time, whereas false recall showed a different pattern, with marked decline in the 2,000ms condition. Separate 2 (identifiability: high vs. low) $\times 3$ (presentation rate: 50 vs. 250 vs. $2,000 \mathrm{~ms}$ ) ANOVAs, with repeated measures over the first factor, were conducted for veridical and false recall. The results of the veridical-recall analysis showed a main effect of presentation rate, $F(2,72)=$ 303.73, MSE $=.006, p<.001, \eta_{\mathrm{p}}{ }^{2}=.89$, and post hoc analyses (Bonferroni tests in all the reported comparisons in the two experiments) revealed that the differences in veridical recall among the three presentation rates were statistically significant in all cases $\left(M_{50 \mathrm{~ms}}=.25\right.$ vs. $M_{250 \mathrm{~ms}}=.39$ vs. $M_{2000 \mathrm{~ms}}=.64$; all $\left.p \mathrm{~s}<.001\right)$. No other main or interaction effects were found for veridical recall.

The results of the false-recall analysis showed a main effect of presentation rate, $F(2,72)=13.80, M S E=.074, p<.001$, $\eta_{\mathrm{p}}{ }^{2}=.28$, with post hoc analyses showing that the slowest presentation rate reliably produced lower levels of false recall $(M=.17)$ than did the $50-\mathrm{ms}$ condition $(M=.40)$ and the 250 -ms condition $(M=.43)$, with $p<.001$ in both cases. The difference in false recall between the 50- and 250-ms conditions was not statistically significant. A significant identifiability $\times$ presentation rate interaction also emerged, $F(2,72)=11.29, M S E=.037, p<.001, \eta_{\mathrm{p}}{ }^{2}=.24$, reflecting the following: (1) In the 50-ms condition, high-identifiable lists reliably produced higher levels of false recall than did low-identifiable lists $(M=.51$ vs. $M=.29 ; p<.001)$; (2) in the 250-ms condition, there was no significant difference between the two types of lists ( $M=.42$ vs. $M=.44)$; and (3) in the 2,000-ms condition, high-identifiable lists reliably

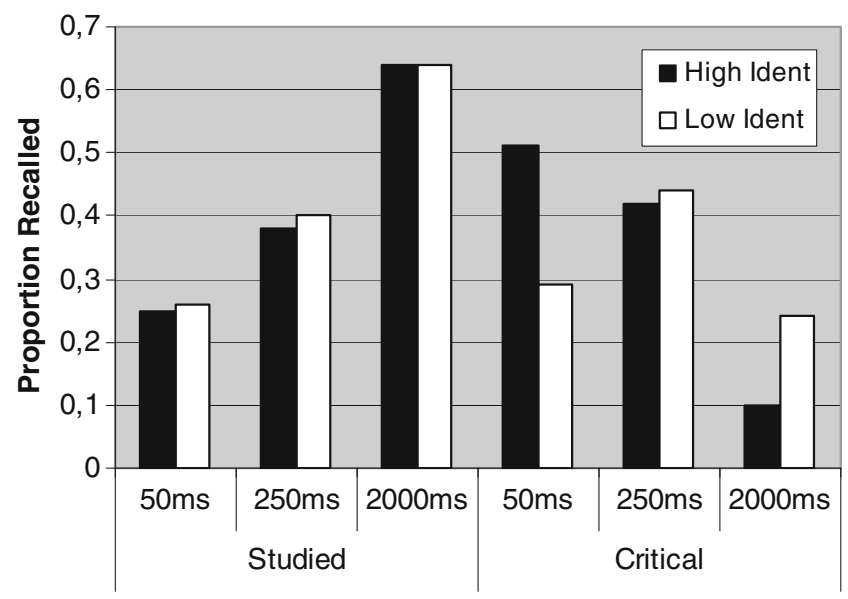

Fig. 1 Proportion of veridical and false recall as a function of presentation rate and identifiability in Experiment 1 
produced lower levels of false recall than did low-identifiable lists $(M=.10$ vs. $M=.24 ; p<.05){ }^{3}$

Recognition task Figure 2 displays the proportion of studied and critical words recognized in each presentation rate condition $(50,250$, and 2,000 ms) for both types of lists (low and high identifiability). The recognition data for 3 participants were not properly registered because of computer problems and, thus, were excluded from the analyses. The overall pattern of recognition data was similar to the pattern observed for recall scores, indicating that exposure duration affects veridical and false recognition in different ways and that identifiability modulates false-memory effects. As is usually the case in studies using the DRM paradigm, false alarms to unrelated distractors were low $\left(M_{50 \mathrm{~ms}}=.02\right.$ vs. $M_{250 \mathrm{~ms}}=.04$ vs. $\left.M_{2000 \mathrm{~ms}}=.02\right)$ and were not significantly affected by the manipulations, while false recognition of critical words reached substantial levels.

Separate 2 (identifiability: high vs. low) $\times 3$ (presentation rate: 50 vs. 250 vs. $2,000 \mathrm{~ms}$ ) ANOVAs, with repeated measures over the first factor, were performed for both veridical and false recognition. The results in veridical recognition showed a main effect of presentation rate, $F(2$, $69)=61.87, M S E=.022, p<.001, \eta_{\mathrm{p}}^{2}=.64$. Similar to what was found in the recall task, the fastest presentation rate $(50 \mathrm{~ms})$ produced the lowest levels of hits, and the slowest presentation rate $(2,000 \mathrm{~ms})$ produced the highest levels of hits, with post hoc comparisons revealing that the three hits means were significantly different from each other $\left(M_{50 \mathrm{~ms}}=.53\right.$ vs. $M_{250 \mathrm{~ms}}=.68$ vs. $M_{2000 \mathrm{~ms}}=.86$; all $p \mathrm{~s}<.001)$. No other main or interaction effects were found for veridical recognition.

In the case of false recognition, the analysis showed a main effect of presentation rate, $F(2,69)=20.81, M S E=.086, p<$. $001, \eta_{\mathrm{p}}{ }^{2}=.38$. The slowest presentation rate produced the lowest level of false recognition $(M=.45)$, significantly inferior $(\mathrm{p}<.001)$ to the levels found in both the 50 -ms condition $(M=.79)$ and the $250-\mathrm{ms}$ condition $(M=.78)$; the later values were almost identical and, unsurprisingly, not

\footnotetext{
${ }^{3}$ Because the difference in concreteness between critical words of lists of high and low identifability approached statistical significance, we decided to perform an additional analysis excluding one list in each group to equate them in the values of this dimension. The two lists that had the highest and lowest values in concreteness were excluded from the high- and low-identifiability groups of lists, respectively, leading to a clearer nonsignificant difference in concreteness $(M=5.77$ vs. $M=$ 5.08), $t(8)=1.29, p=.23$. Performing the same type of analysis without these two lists, the results proved to be very similar, revealing also a significant identifiability $\times$ presentation rate interaction, $F(2$, $72)=12.57, M S E=.043, p<.001, \eta_{\mathrm{p}}{ }^{2}=.26$. Post hoc tests showed the same pattern of higher levels of false recall for high-identifiable lists in the 50 -ms condition $(M=.53$ vs. $M=.31 ; p<.001)$ and lower levels of false recall for these lists in the 2,000-ms condition $(M=.09$ vs. $M=.28 ; p<.01)$.
}

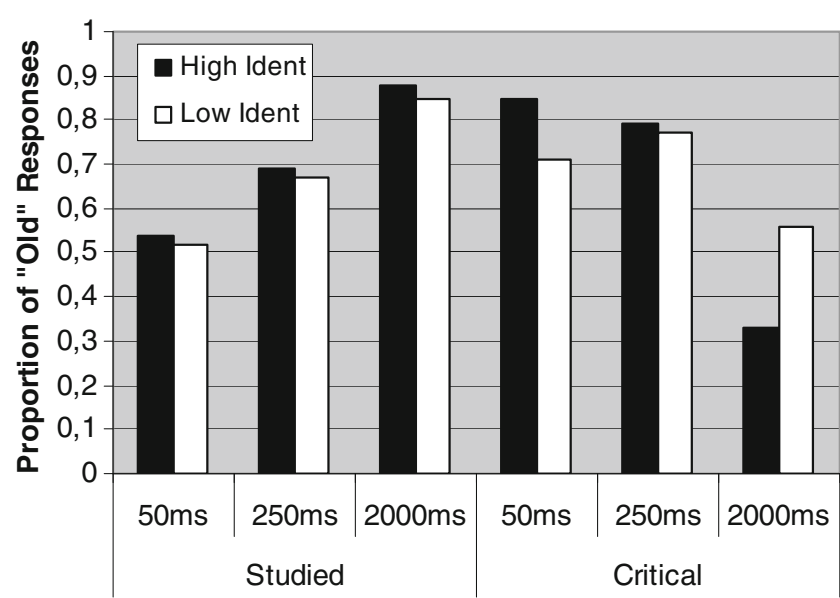

Fig. 2 Proportion of "old" responses in the recognition task for studied and critical words as a function of presentation rate and identifiability in Experiment 1

significantly different from each other. Furthermore, a significant identifiability $\times$ presentation rate interaction was found, $F(2,69)=12.07, \operatorname{MSE}=.035, p<.001, \eta_{\mathrm{p}}{ }^{2}=.26$, revealing the following: (1) In the 50-ms condition, high-identifiable lists produced reliably higher levels of false recognition than did low-identifiable lists $(M=.86$ vs. $M=.71 ; p<.001)$; (2) in the 250-ms condition, there was a nonsignificant difference between the two types of lists ( $M=.79$ vs. $M=.77)$; and (3) in the 2,000-ms condition, high-identifiable lists produced significantly lower levels of false recognition than did lowidentifiable lists $(M=.33$ vs. $M=.56 ; p<.001)$. Similar statistical analyses using signal detection theory ( $A$ ' values) showed the same pattern of results. ${ }^{4}$

In summary, the results were very similar in both memory tasks. It should be pointed out that recognition results should be interpreted with caution because, when the recognition test follows a recall task, a contamination can occur and the recognition results might mimic the recall pattern (Roediger \& McDermott, 1995). However, this does not seem so problematic, since there is also some evidence showing that

\footnotetext{
${ }^{4}$ Veridical recognition of $A$ ' values showed only a main effect of presentation rate, $F(2,69)=43.38, M S E=.002, p<.001, \eta_{\mathrm{p}}{ }^{2}=.56$, with significant increases in veridical recognition as presentation rate increased $\left(M_{50 \mathrm{~ms}}=.85\right.$ vs. $M_{250 \mathrm{~ms}}=.89$ vs. $\left.M_{2000 \mathrm{~ms}}=.95\right)$. For false recognition, there was a main effect of presentation rate, $F(2,69)=$ $16.23, M S E=.009, p<.001, \eta_{\mathrm{p}}{ }^{2}=.32$, with a significant decrease of false recognition between 50 and $2,000 \mathrm{~ms}$ and between 250 and $2,000 \mathrm{~ms}\left(M_{50 \mathrm{~ms}}=.91\right.$ vs. $M_{250 \mathrm{~ms}}=.90$ vs. $\left.M_{2000 \mathrm{~ms}}=.81\right)$. Furthermore, there was also a significant identifiability $\times$ presentation rate interaction, $F(2,69)=13.53, M S E=.003, p<.001, \eta_{\mathrm{p}}{ }^{2}=.28$, reflecting significant differences between high- and low-identifiable lists for the 50- and 2,000-ms conditions, but in opposite directions: In the 50-ms condition, high-identifiable lists produced higher levels of false recognition than did low-identifiable lists $(M=.93$ vs. $M=.89)$, whereas in the 2,000-ms condition, high-identifiable lists produced lower levels of false recognition than did low-identifiable lists $(M=.77$ vs. $M=.85)$.
} 
the interlist variability on recognition level is not compromised by previous recall tests (Brainerd et al., 2008) and that the correlation between the false alarm rates for critical distractors with and without previous recall is usually high (e.g., around .90 in the study by Gallo \& Roediger, 2002).

Veridical recall and recognition increased with item exposure time, regardless of the lists' identifiability. More interesting, and as expected, parallel significant interaction effects were found in false recall and in false recognition. High-identifiable lists produced lower levels of false memories than did low-identifiable lists for the slowest presentation rate but higher levels of false memories for the fastest presentation rate. As was previously hypothesized, these results strongly suggest that, in standard conditions, the participants could reject the false memories provoked by high-identifiable lists but that they were unable to do that when the presentation rate was too fast. In the fast condition, memory rejection was not possible, because the participants could visualize only a few words from the lists but were aware that many other words that they could not see were presented. Hence, they never knew whether the theme word was presented or not, which precluded the possibility of using a memory rejection strategy based on theme identifiability. This could explain why the identifiability effect was eliminated, but not why high-identifiable lists produced more false memories when the presentation rate was fast. The latter finding suggests that even in conditions in which the participants could visualize only a few words from the lists, the themes of high-identifiable lists could be identified and, without the possibility of tagging those words for rejection, more false memories were in the end produced. This result is particularly interesting because it shows that theme identifiability can influence false memories even when it is not possible to perceive all the words in a list.

These results also corroborate previous findings regarding the effect of presentation rates in false memories (e.g., McDermott \& Watson, 2001), consistently showing that, in general, false memories decrease as presentation rates increase. This phenomenon was previously attributed to memory-editing mechanisms that could involve disqualifying monitoring or diagnostic monitoring (Gallo, 2006). The fact that, in the present experiment, the participants were more able to reject false memories when the critical words were more easily identified as the theme of their lists, together with the finding that veridical memory was not affected by identifiability, suggests that the type of memory-editing mechanism that better explains the effect of presentation rate is based more on the identify-to-reject strategy than on diagnostic monitoring. Thus, this experiment has also contributed to understanding how the identify-to-reject strategy can account for the decrease of false memories with prolonged exposure times.

\section{Experiment 2}

In this second DRM experiment, response deadline was manipulated as a between-subjects variable. As was previously argued, the successful application of the identify-to-reject strategy implies retrieving the critical words that have earlier been tagged as unpresented, in order to reject them in a memory test. It is known that whereas familiarity is relatively fast, recollective and monitoring processes are comparatively slow, requiring more time to be successfully applied (McElree et al., 1999; Rotello \& Heit, 2000). Therefore, and according to our prediction, the effect of theme identifiability might be disrupted when retrieval conditions do not facilitate the operation of recollective processes that need time to be operative.

Some other studies have already shown that time pressure at retrieval eliminates some effects that are due to the operation of a memory-editing process. For example, Israel and Schacter (1997) and Schacter et al. (1999) discovered that presenting the associates of a critical word in a distinctive format (pictures instead of words) reduced false recognition, most likely because the participants, relying on a distinctiveness heuristic, could reject the unpresented critical items by detecting the absence of an expected recollection. Interestingly, using similar stimuli and procedures at study, Dodson and Hege (2005) were able to show that pressing participants to produce a response in less than $1 \mathrm{~s}$ eliminated the false-recognition suppression effect that usually results from pictorial encoding. This finding can be interpreted as an indication that speeded retrieval may disable the use of the distinctiveness heuristic, arguably because, as happens with other editing mechanisms, this memory-editing strategy is a timeconsuming process. Another pertinent example comes from a study by Benjamin (2001), which showed that, under standard test conditions, study list repetitions during study decreased false recognition, because multiple study trials facilitate the later use of recollective and decisional processes; however, under time pressure test conditions, the effect of study repetitions was to actually increase false recognition. This contrasting pattern of results can be taken as supporting evidence for a dual-process explanation, in which increased false memory can be attributed to enhanced accessibility to the critical item, fostered by repetitions of the list, in the absence of an opportunity to apply a rejection mechanism because of the unavailability of the necessary time in fast-response situations.

In the present experiment, false recognition of easy- and hard-to-identify critical words was analyzed in two test conditions: self-paced and speeded response. As in many earlier studies of this kind, fast responses in the timeconstrained condition were elicited using the signal-to- 
response technique first introduced by Reed (1973), and they were prompted with a very short delay between the probe and the signal: $250 \mathrm{~ms}$. On the basis of previous evidence regarding both the identifiability effect and the importance of time availability for successful editing of recognition responses, it was expected that in conditions of speeded response, the identifiability effect might be eliminated, to the effect that high- and low-identifiable lists should produce similar levels of false recognition.

\section{Method}

Participants Seventy-two volunteers from Portuguese universities took part in Experiment 2. All were native Portuguese speakers (47 of them female; mean age $=$ 24 years), studying different degree courses (e.g., medicine, psychology, law, math, engineering, biology). They were randomly assigned to either the self-paced or the speeded condition, and they were all tested individually. The participants' ages did not differ significantly between the two conditions, and none of them had taken part in the previous experiment.

Design The experiment followed a 2 (identifiability: high vs. low) $\times 2$ (test condition: self-paced response vs. speeded response) mixed factorial design, with repeated measures over the first factor.

Materials and procedure The same 12 lists as those in Experiment 1 were used in the present experiment, and again, a customized program developed with E-Prime software was utilized to control the sequence of experimental events during the study and test phases. At the beginning of the session, the participants were informed that their task was to memorize lists of words in preparation for a final memory test and to solve interpolated sets of mathematical problems. The study phase was identical in the two conditions, and it required that each participant study a randomly selected set of six associative lists (three of low identifiability and three of high identifiability), with the other half of the lists being reserved for the generation of unrelated distractors to be included in the final recognition test. The 15 words on each list were digitally prerecorded and auditorily presented, at a rate of $1.5 \mathrm{~s} /$ word, The presentation of each list, in an order that was randomly determined for each participant, was followed by a 90-s period in which the participants had to check whether the solutions in a set of mathematical operations displayed on the screen were right or wrong, responding by pressing one of two keys on the computer keyboard.

After the presentation of the six study lists and following the last set of mathematical problems, the participants were instructed to perform the recognition task, in which the words were individually and visually presented at the center of the computer screen and remained there until a response was given. The recognition test for each participant was composed of 48 words, of which 18 words were previously studied associates ( 3 from each studied list, corresponding to items in serial positions 1,6 , and 11), 6 were the critical words corresponding to these studied lists, 18 were new words extracted from the six unpresented lists (again from positions 1, 6, and 11), and 6 were also new words, but this time corresponding to the critical words of the unpresented lists. This choice of distractors was dictated by the goal of having a set of items that could mirror structural characteristics of the target set.

In the self-paced condition, participants were informed that they had the time they wanted to answer "yes" or "no," depending on whether the word had or had not been auditorily presented in the preceding set of to-beremembered lists. In the speeded response condition, the participants were instructed to respond within a very short time, immediately after an acoustic signal sounded. In this condition, the signal was produced with a delay of $250 \mathrm{~ms}$ after the appearance of the word on the screen. If the response was given before the presentation of the acoustic signal or was delayed more than $500 \mathrm{~ms}$ after the signal, the participants were advised by a specific computer message to answer only after the signal or to be faster in the response. Responses given outside these limits were registered but not included in the analyses.

\section{Results}

Figure 3 displays the proportion of recognition for targets, critical words, and unrelated distractors (composed by critical items plus list items from unpresented lists) in both conditions (speeded and self-paced) and with both types of lists (low and high identifiability). First, 2 (identifiability: high vs. low) $\times 2$ (test condition: self-paced response vs. speeded response) mixed factorial ANOVAs, with repeated measures over the first factor, were performed for each type of item. The results of veridical recognition showed a main effect of test condition, $F(1,70)=8.28, M S E=.054, p<$ $.01, \eta_{\mathrm{p}}{ }^{2}=.11$, with the self-paced condition producing higher levels of correct recognition than did the speeded condition $(M=.74$ vs. $M=.62)$. No other significant effects were observed for veridical recognition. The analysis of false recognition scores showed a main effect of test condition, $F(1,70)=5.73, M S E=.138, p<.05, \eta_{\mathrm{p}}{ }^{2}=$ .08 , in which the speeded condition produced higher levels of false recognition than did the self-paced condition $(M=$ .70 vs. $M=.55)$. There was also a significant test condition $\times$ identifiability interaction, $F(1,70)=10.87, M S E=.064$, $p<.01, \eta_{\mathrm{p}}^{2}=.13$. Post hoc analyses showed that in the 


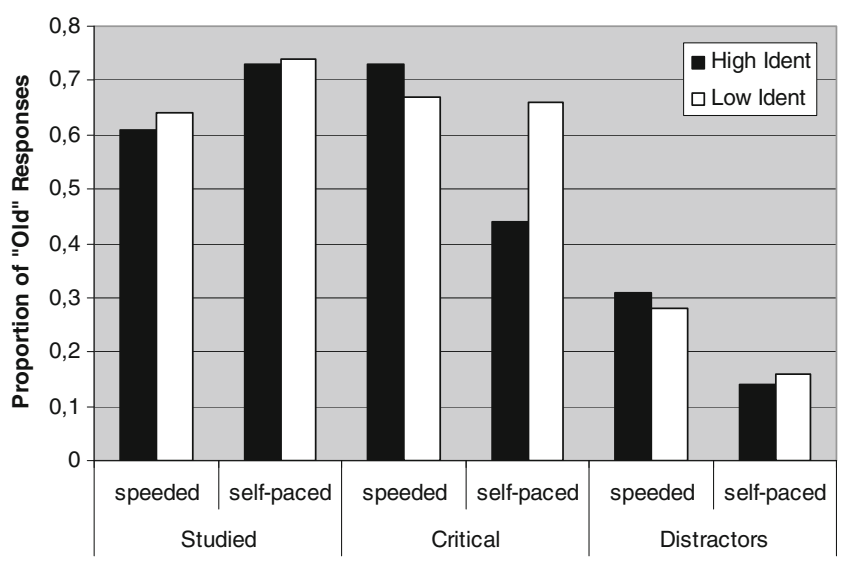

Fig. 3 Proportion of "old" responses in the recognition task for studied, critical, and distractor words as a function of test conditions (self-paced/speeded) and identifiability in Experiment 2

speeded condition, no significant difference existed between the high- and low-identifiability lists $(M=.73$ vs. $M=.67)$, whereas in the self-paced condition, the lists of high identifiability produced lower levels of false recognition than did the lists of low identifiability $(M=.44$ vs. $M=.66$, $p<.01)$. The analysis of unrelated distractors showed only a main effect of test condition, $F(1,70)=10.46, M S E=.071$, $p<.01, \eta_{\mathrm{p}}{ }^{2}=.13$, revealing a higher rate of incorrect recognitions in the speeded condition than in the self-paced condition $(M=.29$ vs. $M=.15)$. No other effects were found. The results were also analyzed by signal detection theory, and a similar pattern was found. ${ }^{5}$

The results of this experiment showed the predicted interaction between identifiability and test condition in the production of false recognition, demonstrating an identifiability effect in a self-paced test condition and the elimination of this effect when very fast recognition responses were required from the participants. On the one hand, the fact that identifiability influenced false recognition only in a self-paced condition suggests that highly identifiable critical words can be rejected by editing processes that require time. On the other hand, the fact that mainly the critical words belonging to highidentifiable lists were rejected suggests that one memoryediting mechanism, most likely of the identify-to-reject type, is operating. As was observed in the previous experiment, the

\footnotetext{
${ }^{5}$ Veridical recognition of $A$ ' values showed only a main effect of test condition, $F(1,70)=16.76, M S E=.042, p<.01, \eta_{\mathrm{p}}{ }^{2}=.09$, indicating that the self-paced condition produced more correct recognition than did the speeded condition $(M=.84$ vs. $M=.70)$. False recognition showed only a significant test condition $\times$ identifiability interaction, $F(1,70)=4.11, M S E=.023, p<.05, \eta_{\mathrm{p}}{ }^{2}=.06$. Post hoc analyses showed that in the speeded condition, no significant difference was found between high- and low-identifiability lists $(M=.71$ vs. $M=$ $.69)$, whereas in the self-paced condition, the lists of high identifiability produced significant lower levels of false recognition than did lists of low identifiability $(M=.66$ vs. $M=.75 ; \mathrm{p}<.05)$.
}

identifiability-related decrement in recognition responses seems to be an effect that occurs specifically for false memories, having no influence on veridical recognition.

\section{General discussion}

This study replicated previous findings by Carneiro et al. (2009), demonstrating a reliable identifiabiliy effect: In standard conditions, lists in which critical words were easily identified as the themes of the lists produced, in general, lower levels of false memories. This effect was replicated not just in conditions involving visual presentation (Experiment 1), but also when lists of associates were auditory presented (Experiment 2). Thus, the effect seems to be robust in the face of modality manipulations, independently of some important semantic dimensions, and once again not limited to conditions in which the participants are explicitly warned about the false-memory phenomenon (cf. Neuschatz et al., 2003).

Furthermore, the results of the present study clarify the mechanisms underlying the identifiability effect, strongly suggesting that this effect is due to the use of one specific memory-editing mechanism - namely, the identify-to-reject strategy. The manipulations in both experiments provided evidence that the effect was eliminated when the conditions, either at encoding or at retrieval, did not allow the successful application of such a strategy. As is indicated by the results of the first experiment, a very fast presentation rate at study disrupted the possibility of noticing that the theme word was not on the list, preventing the action of the strategy during encoding, and as a result, the identifiability effect could no longer be observed. Similarly, as is indicated by the results of the second experiment, forcing the participants to respond quickly disrupted the possibility of using identifiability information during editing at retrieval, and hence, the effect could not occur.

But more interesting, in the fast presentation rate condition of Experiment 1, the results, besides showing the elimination of the identifiability effect, also showed an inversion of the pattern: False memories, in both recall and recognition, were actually higher for high-identifiable lists. It seems that it is not necessary to perceive all the presented items to identify the theme of the list. As other studies have shown, false memories can arise even at such extremely rapid rates as $20 \mathrm{~ms}$ (Cotel, Gallo, \& Seamon, 2008; McDermott \& Watson, 2001; Seamon, Luo, \& Gallo, 1998). In our own experiment, the findings are very consistent with the idea that when it is not possible to detect whether the theme word was in the list or not but the theme word is highly identifiable, high levels of false memories tend to occur. In short, it seems that in a first stage, high identifiability stimulates false memories, but if 
the high identifiable critical words can be tagged as not actually presented, they are more prone to be rejected, and the final result will be lower levels of false memories for those words. This kind of result, showing the action of opposing processes, can be of special theoretical relevance, because it seems to support fuzzy trace theory. Gist identification can increase false memories when memory editing is prevented but can invert the pattern if a rejection process is allowed. In the last case, critical items that come to mind during study can serve as the basis of recollection rejection. Predictions for other theories that explain false memories by spreading activation between preexisting conceptual representations, such as activation monitoring (Roediger, Balota, \& Watson, 2001), were not taken into consideration, since the type of manipulation used in the present study - theme identifiability - seems to be particularly suitable for deriving conclusions about the semantic content of the information (i.e., gist). However, this does not mean that the present results go against the activationmonitoring theory, at least until the relationships between theme identifiability and BAS is better understood.

Although we also found a similar inversion pattern in the speeded test condition of Experiment 2, the difference between low- and high-identifiable lists did not reach statistical significance. Additional research could clarify whether this inversion pattern is a finding likely to be found rather generally, whenever experimental conditions lead to impairment of monitoring strategies, or whether it is a rather specific effect that can be observed only when monitoring strategies are disrupted at encoding.

Gist identification can be viewed as one variable that stimulates false memory but also, and more crucially, as a variable that facilitates memory editing by making salient the item that should be rejected. At first sight, the facilitation of memory editing by theme identifiability, leading to a decrease of false memories in standard conditions, seems to go against the results of think-out-loud studies in which participants are typically asked to say everything they think about while studying a list of items (Lampinen et al., 2005; Lampinen, Ryals, \& Smith, 2008; Seamon, Lee, Toner, Wheeler, Goodkind $\&$ Birch, 2002). Those studies have shown that as more critical words are thought about during encoding, more false memories tend to be produced. However, although the procedure of think-out-loud studies could resemble the procedure used in the identifiability studies, two important differences might explain the opposing results. First, the mere fact that the think-out-loud procedure encourages the initial unrestricted production of many thoughts and words would lead to an increment of potentially interfering material and would pose a difficulty for the operation of an editing mechanism at the time of the test. Second, thinking about the words in the think-out-loud procedure is not the same as identifying the critical word. The critical words generated in the encoding phase in the think-outloud procedure could be critical words of different levels of identifibility but more automatically activated (probably with higher BAS). We do not assume that false memories arise only by theme identifiability. False memories could also be raised by associative activation. Actually, the studies by Seamon et al. (2002) and Lampinen et al. (2005) showed that participants falsely recalled the critical items that were generated in the think-out-loud phase but also that there was a considerable level of false recall for critical items that were not generated. This could mean that false memories can be produced by a generation process that invokes gist representations, but also by a fast-acting automatic activation, which goes in line with this argument. One main message of the present study is, instead, that it is the rejection process that might be affected more by theme identifiability. The identification of the critical word by making the critical word salient might facilitate its rejection. This perspective, that false memories could arise by associative activation and thematic extraction but be rejected mainly by theme identifiability, although speculative, might represent a consensual point of view between associative activation and fuzzy trace theories. In this way, it would be possible to reconcile the identifiability effect, the results of the think-out-loud studies, and the effect of BAS on false memories. However, it is unquestionable that this interpretation needs further empirical support. Only additional studies, conducted with a large set of well-characterized lists, and specifically aiming to analyze the relationship between identifiability, BAS, and false memory production, will start to achieve this goal.

From another perspective, the present study can contribute to better understanding of how the identifyto-reject strategy, as a memory-editing mechanism, works. This strategy, as we conceive it, is composed of several phases, some of which are operating at encoding and others at retrieval. The successful application of this strategy implies, in the encoding phase, that the participants notice that all or most of the words in a list are related to a common theme, that they can identify the theme word that connects all the presented words, and that they realize that this theme word is not being presented in the list. If, for any reason, participants cannot identify the critical word as the converging and missing word from the list, using this memoryediting strategy is not feasible, as usually happens for lists whose critical words are hard to identify as the themes of the lists. Furthermore, the application of this strategy is also dependent on favorable conditions at retrieval. If, for any reason, participants cannot retrieve the identified missing theme from memory at time of 
test, they cannot avoid producing an error. If, on the other hand, the participants are able to retrieve those missing theme words at time of test, they will be able to reject them in the memory test. In sum, this strategy seems to begin at encoding with a correct identification of the theme of the list and with the awareness that the theme word was not presented. But as occurs with other memory-editing mechanisms, this strategy needs time at retrieval to be effective. Thus, differently from what happens in some other memory-editing mechanisms (e.g., the distinctiveness heuristic), identify-to-reject seems to be a memory-editing strategy that does not operate only at retrieval, but starts at encoding and finishes at retrieval.

Another interesting difference observed in comparison with other memory-editing mechanisms is that the identify-to-reject strategy does not seem to be dependent on the level of veridical memory. As the results of both experiments showed, there were no significant differences in correct recall or correct recognition between high- and low-identifiable lists. This means that, in contrast to false memory, veridical memory is not much affected by theme identifiability. When other memoryediting mechanisms are assumed to be in operation, the common pattern is that higher veridical memory is usually tied to lower false memory. For example, in the case of the distinctiveness heuristic, the presentation of more distinctive associates (pictures) usually produces lower levels of false memory but higher levels of veridical recall (Israel \& Schacter, 1997). And even in other instances of recollection rejection or disqualifying monitoring, this veridical-memory dependence seems to occur. Gallo (2006) referred to source-based exclusions as one way of using the recall-to-reject process, in which the recall of a specific item in one context is mutually exclusive with its occurrence in another context. Moreover, another specific case of the recall-to-reject process is to use the exhaustive-recall-to-reject strategy. If there is an exhaustive recall of all the studied items, this perfect, correct recall necessarily leads to null false recall. Thus, for the other memory-editing strategies, false memory seems to be negatively correlated with veridical memory, whereas for the identify-to-reject strategy, false memory seems to have no correlation with veridical memory. This could suggest that this strategy does not rely on memory for studied items but, instead, can be assumed to rely on memory of the critical distractor coming to mind during study, a memory that could later serve as the basis of monitoring decisions such as disqualifying monitoring or recollection rejection.

Even from a cursory scrutiny of the vast literature showing effects of meaning and organization on memory (e.g., Bower, Clark, Lesgold, \& Winzenz, 1969; Mandler,
1967), this null result of identifiability on veridical memory may seem odd. Nevertheless, it should be noted that in the case of the DRM paradigm, all lists are organized and constructed by meaning. It is obvious for any participant that the words on each list are all related to a common theme, and it is well known that relational processing usually increases veridical memory (Hunt \& Einstein, 1981). However, the identification of the theme does not seem to be important for veridical memory. Even without knowing what the theme is, the presentation of words that are related to each other will increase veridical memory. On the contrary, in the case of false memory, identification of the critical item seems to be the key for successful monitoring. This seems to explain the apparent discrepancy in the results between these studies and why theme identifiability did not affect veridical memory but affected false memory.

In sum, the present results, together with previous findings in this line of research (Carneiro et al., 2009), indicate that theme identification plays a significant role in the rejection of false memories in the DRM paradigm and that the identify-to-reject strategy is the memory-editing mechanism responsible for the identifiability effect. We have shown that in standard conditions, the identifiability effect is observed, but when the identify-to-reject strategy cannot be operative, due to specific manipulations either at encoding or at retrieval, this effect is disrupted. We hope to have contributed to emphasizing the importance of theme identifiability for understanding the mechanisms behind false memory formation when measured by converging associative tasks and to highlighting the contribution of the identify-to-reject strategy, as one of the memory-editing mechanisms that operates in DRM tasks.

\section{References}

Albuquerque, P. B. (2001). Normas de associação semântica de palavras portuguesas para aplicar ao paradigma DRM. Braga: Relato Técnico, Universidade do Minho.

Benjamin, A. S. (2001). On the dual effects of repetition on false recognition. Journal of Experimental Psychology: Learning, Memory, and Cognition, 27, 941-947.

Bower, G. H., Clark, J. C., Lesgold, A. M., \& Winzenz, D. (1969). Hierarchical retrieval schemes in recall of categorical word lists. Journal of Verbal Learning and Verbal Behavior, 8, 323343.

Brainerd, C. J., \& Reyna, V. F. (2005). The science of false memory. New York: Oxford University Press.

Brainerd, C. J., Reyna, V. F., Wright, R., \& Mojardin, A. H. (2003). Recollection rejection: False memory editing in children and adults. Psychological Review, 110, 762-784.

Brainerd, C. J., Wright, R., Reyna, V. F., \& Payne, D. G. (2002). Dualretrieval processes in free and associative recall. Journal of Memory and Language, 46, 120-152. 
Brainerd, C. J., Yang, Y., Reyna, V. F., Howe, M. L., \& Mills, B. A. (2008). Semantic processing in "associative" false memory. Psychonomic Bulletin \& Review, 15, 1035-1053.

Brédart, S. (2000). When false memories do not occur: Not thinking of the lure or remembering that it was not heard? Memory, 8, $123-128$.

Brown, J., Lewis, V. J., \& Monk, A. F. (1977). Memorability, word frequency and negative memory. Quarterly Journal of Experimental Psychology, 29, 461-473.

Cann, D. R., McRae, K., \& Katz, A. N. (2011). False recall in the Deese-Roediger-McDermott paradigm: The roles of gist and associative strength. Quarterly Journal of Experimental Psychology, 64, 1515-1542.

Carneiro, P., Fernandez, A., \& Dias, A. R. (2009). The influence of theme identifiability on false memories: Evidence for agedependent opposite effects. Memory \& Cognition, 37, 115129 .

Cotel, S. C., Gallo, D. A., \& Seamon, J. G. (2008). Evidence that nonconscious processes are sufficient to produce false memories. Consciousness and Cognition, 17, 210-218.

Deese, J. (1959). On the prediction of occurrence of particular verbal intrusions in immediate recall. Journal of Experimental Psychology, 58, 17-22.

Dodson, C. S., \& Hege, A. C. G. (2005). Speeded retrieval abolishes the false memory suppression effect: Evidence for the distinctiveness heuristic. Psychonomic Bulletin \& Review, 12, 726-731

Dodson, C. S., \& Schacter, D. L. (2001). "If I had said it I would have remembered it": Reducing false memories with a distinctiveness heuristic. Psychonomic Bulletin \& Review, 8, 155-161.

Dodson, C. S., \& Schacter, D. L. (2002). When false recognition meets metacognition: The distinctiveness heuristic. Journal of Memory and Language, 46, 782-803.

Gallo, D. A. (2006). Associative illusions of memory: False memory research in DRM and related tasks. New York: Psychology Press.

Gallo, D. A. (2010). False memories and fantastic beliefs: 15 years of the DRM illusion. Memory \& Cognition, 38, 833-848.

Gallo, D. A., \& Roediger, H. L. (2002). Variability among word lists in eliciting memory illusions: evidence for associative activation and monitoring. Journal of Memory and Language, 47, 469497.

Gallo, D. A., Roberts, M. J., \& Seamon, J. G. (1997). Remembering words not presented in lists: Can we avoid creating false memories? Psychonomic Bulletin \& Review, 4, 271-276

Hunt, R. R., \& Einstein, G. O. (1981). Relational and item-specific information in memory. Journal of Verbal Learning and Verbal Behavior, 20, 497-514.

Israel, L., \& Schacter, D. L. (1997). Pictorial encoding reduces false recognition of semantic associates. Psychonomic Bulletin \& Review, 4, 577-581.

Jacoby, L. L. (1991). A process dissociation framework: Separating automatic from intentional uses of memory. Journal of Memory and Language, 30, 513-541.

Lampinen, J. M., Meier, C. R., Arnal, J. D., \& Leding, J. K. (2005). Compelling untruths: Content borrowing and vivid false memories. Journal of Experimental Psychology: Learning, Memory, and Cognition, 31, 954-963.

Lampinen, J. M., Ryals, D. B., \& Smith, K. (2008). Compelling untruths: The effect of retention interval on content borrowing and vivid false memories. Memory, 16, 149-156.

Madigan, S., \& Neuse, J. (2004). False recognition and word length: A reanalysis of Roediger, Watson, McDermott, and Gallo (2001) and some new data. Psychonomic Bulletin \& Review, 11, 567573 .
Mandler, G. (1967). Organization and memory. In K. W. Spence \& J. T. Spence (Eds.), The psychology of learning and motivation: Advances in research and theory (Vol. 1, pp. 328-372). New York: Academic Press.

McCabe, D. P., Presmanes, A. G., Robertson, C. L., \& Smith, A. D. (2004). Item-specific processing reduces false memories. Psychonomic Bulletin \& Review, 11, 1074-1079.

McDermott, K. B. (1996). The persistence of false memories in list recall. Journal of Memory and Language, 35, 212-230.

McDermott, K. B., \& Watson, J. M. (2001). The rise and fall of false recall: The impact of presentation duration. Journal of Memory and Language, 45, 160-176.

McElree, B., Dolan, P. O., \& Jacoby, L. L. (1999). Isolating the contributions of familiarity and source information to item recognition: A time-course analysis. Journal of Experimental Psychology: Learning, Memory, and Cognition, 25, 563-582.

Mukai, A. (2005). Awareness of the false memory manipulation and false recall for people's names as critical lures in the DeeseRoediger-McDermott paradigm. Perceptual and Motor Skills, 101, 546-560.

Nascimento, M. F. B., Casteleiro, J. M., Marques, M. L. G., Barreto, F., \& Amaro, R. (n.d.). Léxico multifuncional computorizado do português contemporâneo. Retrieved from www.clul.ul.pt/index.php

Nelson, D. L., McEvoy, C. L., \& Schreiber, T. A. (2004). The University of South Florida free association, rhyme, and word fragment norms. Behavior Research Methods, Instruments, \& Computers, 36, 402-407.

Neuschatz, J. S., Benoit, G. E., \& Payne, D. G. (2003). Effective warnings in the Deese-Roediger-McDermott false-memory paradigm: The role of identifiability. Journal of Experimental Psychology: Learning, Memory, and Cognition, 29, 35-41.

Pérez-Mata, M. N., Read, J. D., \& Diges, M. (2002). Effects of divided attention and word concreteness on correct recall and false memory reports. Memory, 10, 161-177.

Pesta, B. J., Murphy, M. D., \& Sanders, R. E. (2001). Are emotionally charged lures immune to false memory? Journal of Experimental Psychology: Learning, Memory, and Cognition, 27, 328-338.

Reed, A. V. (1973). Speed-accuracy trade-off in recognition memory. Science, 181, 574-576.

Roediger, H. L., III, Balota, D. H., \& Watson, J. M. (2001). Spreading activation and the arousal of false memories. In H. L. Roediger III, J. S. Nairne, I. Neath, \& A. M. Surprenant (Eds.), The nature of remembering: Essays in honor of Robert G. Crowder (pp. 95-115). Washington, DC: American Psychological Association.

Roediger, H. L., III, \& McDermott, K. B. (1995). Creating false memories: Remembering words not presented in lists. Journal of Experimental Psychology: Learning, Memory, and Cognition, 21, 803-814.

Roediger, H. L., III, Watson, J. M., McDermott, K. B., \& Gallo, D. A. (2001). Factors that determine false recall: A multiple regression analysis. Psychonomic Bulletin \& Review, 8, 385-407.

Rotello, C. M., \& Heit, E. (2000). Associative recognition: A case of recall-to-reject processing. Memory \& Cognition, 28, 907922.

Schacter, D. L., Cendan, D. L., Dodson, C. S., \& Clifford, E. (2001). Retrieval conditions and false recognition: Testing the distinctiveness heuristic. Psychonomic Bulletin \& Review, 8, $827-833$.

Schacter, D. L., Israel, L., \& Racine, C. (1999). Suppressing false recognition: The distinctiveness heuristic. Journal of Memory and Language, 40, 1-24.

Seamon, J. G., Lee, I. A., Toner, S. K., Wheeler, R. H., Goodkind, M. S., \& Birch, A. D. (2002). Thinking of critical words during 
study is unnecessary for false memory in the DRM procedure. Psychological Science, 13, 526-531.

Seamon, J. G., Luo, C. R., \& Gallo, D. A. (1998). Creating false memories of words with or without list item recognition: Evidence for nonconscious processes. Psychological Science, 9, 20-26.

Thapar, A., \& McDermott, K. B. (2001). False recall and false recognition induced by presentation of associated words: Effects of retention interval and level of processing. Memory \& Cognition, 29, 424-432.

Toglia, M. P., \& Battig, W. F. (1978). Handbook of semantic word norms. Hillsdale: Erlbaum.
Wu, L. L., \& Barsalou, L. W. (2009). Perceptual simulation in conceptual combination: Evidence from property generation. Acta Psychologica, 132, 173-189.

The work of Paula Carneiro was supported by the Portuguese Foundation of Science and Technology (Project PTDC/PSI-PCO/ 112466/2009). The work of Angel Fernandez and Emiliano Diez was supported by the Spanish Ministry of Science and Innovation (Project PSI2008-05607/PSIC) 